\title{
In vivo monitoring of bone microstructure by propagation-based phase-contrast computed tomography using monochromatic synchrotron light
}

\author{
Takeshi Matsumoto $^{1} \cdot$ Ryota Shimizu $^{1} \cdot$ Kentaro Uesugi $^{2}$
}

Received: 10 May 2019 / Revised: 3 October 2019 / Accepted: 3 October 2019 / Published online: 22 October 2019

(c) The Author(s), under exclusive licence to United States and Canadian Academy of Pathology 2019

\begin{abstract}
Hard X-ray phase-contrast imaging is sensitive to density variation in objects and shows a dose advantage for in vivo observation over absorption-contrast imaging. We examined the capability of propagation-based phase-contrast tomography (PB-PCT) with single-distance phase retrieval for tracking of bone structure and mineral changes using monochromatic synchrotron light. Female mice underwent ovariectomy and drill-hole surgery in the right tibial diaphysis and were divided into two groups: OVX and OVX-E ( $n=6$ each); the latter group was treated with intraperitoneal administration of 14,15epoxyeicosatrienoic acid (14,15-EET) for promoting bone repair. Age-matched mice subjected to sham ovariectomy and drill-hole surgery (Sham) were also prepared $(n=6)$. In vivo CT scans of the drilled defect were acquired 3, 7, and 11 days after surgery, and tomographic images were matched by three-dimensional registration between successive time points for monitoring the process of defect filling. In addition, using absorption-contrast $\mathrm{CT}$ as the reference method, the validity of PB-PCT was evaluated in one mouse by comparing images of tibial metaphyseal bone between the two methods in terms of bone geometry as well as the measure of mineralization. Although phase retrieval is strictly valid only for single-material objects, PB-PCT, with its lower radiation dose, could provide a depiction of bone structure similar to that from absorptioncontrast CT. There was a significant correlation of linear absorption coefficients between the two methods, indicating the possibility of a rough estimate of the measure of mineralization by PB-PCT. Indeed, delayed bone regeneration (OVX vs. Sham) and the efficacy of 14,15-EET for improving osteoporotic bone repair (OVX-E vs. OVX) could be detected in both bone volume and mineralization by PB-PCT. Thus, in combination with single-distance phase retrieval, PB-PCT would have great potential for providing a valuable tool to track changes in bone structure and mineralization, and for evaluating the effects of therapeutic interventions as well.
\end{abstract}

\section{Introduction}

$\mathrm{X}$-ray computed tomography (CT) is most frequently used for the measurement of bone microarchitecture, allowing

Supplementary information The online version of this article (https:// doi.org/10.1038/s41374-019-0337-3) contains supplementary material, which is available to authorized users.

Takeshi Matsumoto

t.matsumoto@tokushima-u.ac.jp

1 Biomedical Engineering Laboratory, Tokushima University Graduate School of Technology, Industrial and Social Sciences, Tokushima 770-8506, Japan

2 SPring-8/Japan Synchrotron Radiation Research Institute, Kouto 679-5198, Japan the assessment of its strength or the efficacy of treatments [1]. More sophisticated imaging has lowered the barriers for its application to in vivo bone imaging, especially in rodent models. In vivo CT was first introduced by Kinney et al. [2] for imaging of rat trabecular bone using monochromatic synchrotron light, and thereafter, laboratory in vivo $\mathrm{CT}$ came into widespread use for bone structural analysis [3].

The use of in vivo CT with either synchrotron radiation or micro-focused X-ray sources enables tracking of 3D bone structure in the same animal, which increases the ability to detect causal relationships and reduces the number of animals needed. High-resolution in vivo CT allows for tracking local bone dynamics and identifying the loci of bone formation and resorption at the single-trabeculum level [4-7]. Computational biomechanical analyses also demonstrate that monitoring of bone structural changes over a long 
period of time may provide valuable information for predicting fracture risk and treatment planning $[8,9]$.

These advantages of in vivo CT are, however, not available without limitations. Repeated scanning results in increased total dose, possibly exerting a substantial impact on bone turnover $[10,11]$. Reducing the total dose is not difficult, but there is a trade-off between radiation dose and spatial resolution or image quality. Far from absorption edges, the dose is inversely proportional to the fourth power of voxel size when the signal-to-noise ratio (SNR) is kept constant; while SNR is linearly related to the square root of dose under the same resolution $[12,13]$. Thus, in vivo CT with high-resolution inevitably exposes bone and surrounding tissues to high doses of radiation.

Increasing the dose efficiency by exploiting the X-ray phase shift or refraction could be a promising way to reduce the dose in X-ray imaging. The X-ray refractive index $n n$, characterizing the optical properties of the material is expressed as $n=1-\delta+i \beta$, in which $\delta$ and $\beta$ characterize the phase shifting and absorption properties of the material, respectively. There is little practical difference in $\beta$ between soft tissues and water $\left(\sim 10^{-10}\right)$. That is, soft tissues are almost transparent to hard X-rays and their absorption contrast is almost invisible. In contrast, $\delta$ is typically three orders of magnitude larger than $\beta$, and therefore, the phase shift-based contrast is excellent even in imaging of soft tissues in which the different components show almost negligible, as well as too similar, absorption to be discriminated [14]. Bone is much less transparent than soft tissues, and conventional absorption-contrast CT works well in bone imaging. However, $\delta$ is still larger than $\beta$ in bone (e.g., $\delta / \beta=300-900$ for cortical bone over $20-40$ $\mathrm{keV})$ [15], and thus, phase shift-based CT may also have a significant dose advantage over absorption-contrast $\mathrm{CT}$ in bone imaging.

Several techniques have been developed, such as propagation-, analyzer-, and interferometry-based imaging methods, to visualize the $\mathrm{X}$-ray phase shift $[15,16]$. Among these, single-distance propagation-based imaging [17, 18], which requires no specific optics system and needs only a single X-ray image per projection angle for tomographic reconstruction, is the simplest and favorable way to reduce dose. By taking a certain distance between an object and the detector, a monochromatic X-ray beam with a plane-wave front can generate the transmission images that contain contrast from attenuation and phase shift through Fresnel diffraction yielded through the object. These images are often processed by a phase retrieval algorithm prior to tomographic reconstruction. Phase retrieval leads to highcontrast visualization with tolerance to noise [19-21], thereby allowing simple thresholding to be used in segmentation of tomographic image volumes. Several studies have proven the benefits of propagation-based imaging with single-distance phase retrieval in CT of bone and other biological tissues [22-27]. To the best of our knowledge, no study has explored its potential for CT tracking of local changes in bone architecture over time.

We tested the feasibility and validity of propagationbased imaging with single-distance phase retrieval in CT tracking of bone microstructure. Synchrotron radiation, providing a high-flux, monochromatic X-ray beam with a high degree of transverse coherence, was employed for single-distance propagation-based imaging, and the process of bone-defect repair in osteoporotic mice and the effect of a promoter of organ regeneration $(14,15$-epoxyeicosatrienoic acid (EET)) [28] on it were monitored. Comparison was also made between tomographic images obtained via propagation-based imaging with single-distance phase retrieval and conventional absorption imaging; the latter imaging is superior in terms of mineral quantitation [5, 29].

\section{Materials and methods}

Experiments were carried out at the Experimental Animal Facility and Biomedical Imaging Center of the SR facility "Super Photon ring-8GeV (SPring-8)" in Japan. The protocol of the animal treatment was in accordance with the guiding principles of the American Physiological Society and with the approval of the Animal Research Committee of Tokushima University and Japan Synchrotron Radiation Research Institute.

\section{Animal preparation}

Female C57BL/6 mice, with either bilateral ovariectomies $(n=12)$ or sham-operated $(n=6)$ at the age of 11 weeks, were purchased from CLEA Japan (Tokyo, Japan). Rodent models of postmenopausal osteoporosis following ovariectomy have been widely used as a model of impaired fracture healing [30, 31]. Mice were single-housed in a plastic cage under controlled conditions (12-h light/dark cycle, $25^{\circ} \mathrm{C}, 60 \%$ humidity) and allowed free access to a standard diet (CLEA Japan) and tap water.

At the age of 12 weeks, the mice were anesthetized with isoflurane, and the skin over the medial aspect of the right lower leg was shaved, swabbed with povidone iodine, and incised. A full-thickness unicortical hole through the upper tibial diaphyseal cortex was created $\sim 3 \mathrm{~mm}$ proximal to the tibio-fibular junction using a $0.5-\mathrm{mm}$ diameter drill rotating at $11,000 \mathrm{rpm}$ (Muromachi Kikai, Kyoto, Japan). Drill margins were frequently irrigated with saline to avoid thermal necrosis, and the drill hole was rinsed with a flushing syringe to discard bone fragments. After arrest of bleeding from the bone marrow, the skin was sutured and swabbed with povidone again. 
Immediately after the drill-hole surgery, the mice were implanted intraperitoneally with a mini-osmotic pump (Alzet model 1002; Cupertino, California, USA). The pump was filled with 14,15-EET, purchased from Cayman Chemical (Ann Arbor, Michigan, USA) or with vehicle (ethanol). Half of the post-ovariectomy mice $(\mathrm{OVX}+\mathrm{E}, n=6)$ received 14,15-EET at a dose of $15 \mu \mathrm{g} / \mathrm{kg} / \mathrm{d}$ [28]. The remaining ovariectomized mice (OVX, $n=6$ ) and the sham-operated mice (Sham, $n=6$ ) received only the delivery of vehicle at an equal volume rate. We administered 14,15-EET in anticipation of improving the defect repair in OVX [30, 31] via its capability to stimulate angiogenesis [32], which is essential for bone healing [33] but reduced in OVX [31]. Soon after they recovered from the general anesthesia, all mice moved around normally.

\section{Propagation-based imaging and CT scanning in vivo}

The drill-hole healing was observed in the experimental hutch $3\left(25 \pm 1^{\circ} \mathrm{C}\right)$ of beamline $20 \mathrm{~B} 2$ at the SPring- 8 synchrotron radiation source (Harima, Japan). A bending magnet source with a $\mathrm{Si}(111)$ double-crystal monochromator, located $\sim 210 \mathrm{~m}$ upstream of the hutch, provided a $300 \mathrm{~mm}$ (horizontal) $\times 20 \mathrm{~mm}$ (vertical) monochromatic $\mathrm{X}$-ray beam for projecting the image of objects onto the detector [34]. The incident X-ray energy was set at 33.4 $\mathrm{keV}$. In a study of in vivo phase-contrast $\mathrm{CT}$ for rat bone imaging [25], the energy of $37 \mathrm{keV}$ proved feasible. Instead, we chose the energy immediately above the k-edge of iodine. The vertical beam size of the incident X-ray was reduced to $2 \mathrm{~mm}$ by a slit built into the beamline end.

We used a visible light conversion type $\mathrm{X}$-ray detector composed of a scientific CMOS (sCMOS) camera (C1144022CU; Hamamatsu Photonics, Hamamatsu, Japan) and a beam monitor (BM2; Hamamatsu Photonics) with a 10- $\mu$ mthick phosphor screen $\left(\mathrm{Gd}_{2} \mathrm{O}_{2} \mathrm{~S}: \mathrm{Tb}^{+}\right)$. The effective pixel size and field of view were $6.54 \mu \mathrm{m}$ and $13.4 \mathrm{~mm}$ (horizontal) $\times 2.0 \mathrm{~mm}$ (vertical), respectively. The distance between the irradiated hindlimb and the detector $(R)$ was set to $60 \mathrm{~cm}$ for propagation-based imaging. With this setting, the minimal Fresnel number $\left(d^{2} / \lambda R\right)[21]$ and the width of the first Fresnel zone $(\sqrt{\lambda R / M})$ [35] were 1.92 and 4.72 $\mu \mathrm{m}$, respectively, where $d$ is the characteristic size of the smallest discernible features in the object, $\lambda\left(3.71 \times 10^{-9} \mathrm{~cm}\right.$ here) is the X-ray wavelength, and $M$ is the geometric magnification, being $\approx 1$ for the plane-wave $\mathrm{X}$-ray. Here, we equated $d$ with the pixel size.

On days 3, 7, and 11 after drill-hole surgery, each mouse was anesthetized by the intraperitoneal administration of pentobarbital sodium and immobilized on a stack of computer-controlled precision stages for CT scanning. The forelimbs and the intact hindlimb were secured with surgical tape in the supine position, and the other hindlimb that

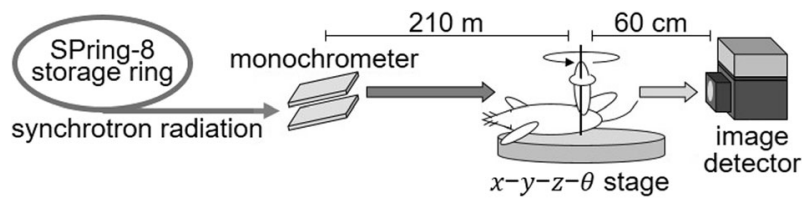

Fig. 1 Experimental setup. An X-ray energy of $33.4 \mathrm{keV}$ was selected using a double bounce monochromator. An anesthetized mouse is placed on a stack of positioning stages in the supine position with stretching the hindlimb subjected to the drill-hole surgery in the vertical direction. The image detector comprises a scientific CMOS camera and a beam monitor with a gadolinium phosphor screen

had undergone the drill-hole surgery was stretched vertically and fixed to an acrylic supporting strut using surgical tape. This posture ensured a roughly proper placement of the drill hole for scanning within the limited area of irradiation. Experimental setup is shown in Fig. 1.

After fine-tuning the vertical position of the drill hole, CT scanning was carried out with propagation-based imaging. We first acquired 30 images each of the sCMOS dark current and direct beam; subsequently, 600 drill-hole images over an angular range of $180^{\circ}$ in $0.3^{\circ}$ steps and 30 images of the direct beam were then acquired. The exposure time per projection angle was set to $100 \mathrm{msec}$. The dose per scan, measured using an ionization chamber (S-1194A1; Ohyo Koken Kogyo, Tokyo, Japan), amounted to 2.0 Gy.

\section{Single-distance phase retrieval and image reconstruction}

Following correction for the dark current and normalization with the direct beam images, the bone images were processed by phase retrieval for generating contact images, which could serve as an equivalent to the images of transmission X-ray intensity taken with the object as close as possible to the detector. We used a well-accepted approach for phase retrieval, originally derived by Paganin et al. [17] on the basis of the transport of intensity equation [36] for a paraxial monochromatic wavefield, assuming that an irradiated object is homogeneous $(\delta / \beta=\gamma=$ constant). This method is available to the objects with nonnegligible absorption like bone tissues. Under the near field approximation [21], the following equation can be obtained using the transport of intensity equation with the $z$ axis in the direction of the incident X-rays:

$I_{R}\left(\boldsymbol{r}_{\perp}\right)=\left[1-\frac{\gamma R}{2 k} \nabla_{\perp}^{2}\right] I_{0}\left(\boldsymbol{r}_{\perp}\right)$

here, $I_{0}\left(\boldsymbol{r}_{\perp}\right)$ and $I_{R}\left(\boldsymbol{r}_{\perp}\right)$ are distributions of X-ray intensity at $z=0$ (contact image) and at $z=R$ (propagation-based image), respectively; $\boldsymbol{r}_{\perp}=(x, y)$ is the position vector lying in the plane perpendicular to the $z$ axis; $\nabla_{\perp}$ is the gradient 
operator in the plane containing $\boldsymbol{r}_{\perp}$; and $k(=2 \pi / \lambda)$ is the wavenumber. Equation 1 can be solved for $I_{0}\left(\boldsymbol{r}_{\perp}\right)$ as

$I_{0}\left(\boldsymbol{r}_{\perp}\right)=\mathcal{F}^{-1}\left\{\frac{1}{\gamma R\left|\mathbf{k}_{\perp}\right|^{2} / 2 k+1} \mathcal{F}\left[I_{R}\left(\boldsymbol{r}_{\perp}\right)\right]\right\}$,

where $\mathcal{F}$ and $\mathcal{F}^{-1}$ are the two-dimensional (2D) forward and inverse Fourier transforms with respect to $\boldsymbol{r}_{\perp}$, and $\mathbf{k}_{\perp}=$ $\left(k_{x}, k_{y}\right)$ is the Fourier coordinate corresponding to $\boldsymbol{r}_{\perp}$. Thus, $I_{0}\left(\boldsymbol{r}_{\perp}\right)$ can be obtained from $I_{R}\left(\boldsymbol{r}_{\perp}\right)$ measured by the detector placed at $z=R$.

In homogeneous objects, both $\delta$ and $\beta$ could be considered proportional to electron density when X-ray energies are well away from the absorption edges of the object; thus, the assumption of constant $\gamma$ is feasible. However, the present object is composed of bone and soft tissues, and furthermore, the bone tissue itself is an approximately threecomponent composite material comprising hydroxyapatitelike mineral, organic constituents (mainly collagen fibrils), and water. Thus, $\gamma$ varies from region to region, and in accordance with the degree of deviation of $\gamma$ from the assumed constant $\gamma$ value, Eq. 2 yields some errors. Nevertheless, in practice, this method shows substantial tolerance to violation of the constant $\gamma$ assumption and its feasibility at least for 3D structure analysis [37]. The method has already demonstrated its high potential for various biological objects comprising different materials, including air, interstitial fluid, soft tissue, and bone [19-27].

The term $\left(\gamma R\left|\mathbf{k}_{\perp}\right|^{2} / 2 k+1\right)^{-1}$ in Eq. 2 is considered as a Lorentzian low-pass filter, and $\gamma$ could be viewed as the filtering parameter, modulating the level of image noise and the blurring at tissue interfaces. Using the $\gamma$ value of bone causes over-smoothing artifact in reconstructing the bone boundary because of soft tissue (mostly muscle and marrow tissues) surrounding the bone tissue. The suitable $\gamma$ for reconstructing the bone/soft-tissue interface may be given by $\left(\delta_{b}-\delta_{s}\right) /\left(\beta_{b}-\beta_{s}\right)[38,39]$, where the subscripts $b$ and $s$ denote bone and soft tissues, respectively. Approximating the values of $\delta_{b}$ and $\beta_{b}$ using the values of fully-mineralized bone, composed of $50 \%$ hydroxyapatite, $40 \%$ collagen, and $10 \%$ water by volume, and the values of $\delta_{s}$ and $\beta_{s}$ using those of water, we used Eq. 2 with $\gamma=3.34 \times 10^{2}$ in the present study (for details, see the Supplementary material).

Tomographic reconstruction from a series of contact images generated by single-distance phase retrieval, here referred to as propagation-based phase-contrast $\mathrm{CT}$ (PB-PCT), was made using a 2D filtered back-projection algorithm. The contiguous sections containing the drill hole were composed of 6.54- $\mu$ m cubic voxels with a resolution of 8-bit gray scale proportional to the linear absorption coefficient $\mu\left(\mathrm{cm}^{-1}\right)$. All reconstructed images were redigitized such that $\mu=0.0106 \times$ gray value $\left(0.0 \mathrm{~cm}^{-1} \leq \mu \leq 2.7 \mathrm{~cm}^{-1}\right)$.

\section{PB-PCT versus absorption-contrast CT}

When using a monochromatic X-ray, absorption-contrast CT is of great advantage when translating its reconstructed image into the distribution of $\mu$. For comparison of CT quantitation, in one mouse from the sham-ovariectomized group, the proximal right tibial region was scanned in vivo by the same system as that used for bone-defect imaging, setting the object-to-detector distance to 60 and $10 \mathrm{~cm}$ for PB-PCT and absorption-contrast CT, respectively. The energy of synchrotron light, the exposure time per projection angle, the method for tomographic reconstruction, and the gray-level normalization were the same as those used in bone-defect imaging. In absorption-contrast CT, 1800 projections were collected in $0.1^{\circ}$ increments to increase the quality of the tomographic images, resulting in a total surface dose of $6 \mathrm{~Gy} / \mathrm{scan}$, three times higher than that in PBPCT.

For quantitative evaluation of mineralization, capillary tubes containing $\mathrm{K}_{2} \mathrm{HPO}_{4}$ water solution at various concentrations were scanned by absorption-contrast $\mathrm{CT}$ in the same manner as the animal imaging, and the relation between the solution density and $\mu$ was determined. Here, it is assumed that bone tissue is uniform and that its absorption can be represented by a two-phase mixture of hydroxyapatite and other light elements, replaced by $\mathrm{K}_{2} \mathrm{HPO}_{4}$ and water, respectively [5, 29]. Thus, the $\mathrm{K}_{2} \mathrm{HPO}_{4}$ solution could serve as a bone phantom and its density is considered as the measure of apparent mineralization. The same solutions were also scanned by PBPCT with the same setting as the animal imaging, and a value of $\mu$ was determined by setting $\gamma=3.34 \times 10^{2}$ for each solution.

\section{Image registration}

A 3D registration technique based on mutual information measure [40] was used for matching 3D images at different time points (PB-PCT images of the cortical defect at different healing stages) or those obtained by different methods (images of the tibial metaphysis obtained by PB-PCT and conventional absorption-contrast CT). The mutual information was calculated while rotating and translating the one image (floating image) with fixing the other (reference image), and the highest degree of image matching was achieved by maximizing the mutual information. Trilinear partial volume distribution interpolation [40] was used when the voxel positions in a floating image (orientation changed) did not coincide with those in the reference image (orientation fixed). When matching of cortical defect images at three healing stages, the image at the second scan was set as the reference. 


\section{Bone image analysis}

Simple thresholding was used to segment tomographic images into bone and non-bone regions. Bone images were compared between PB-PCT and absorption-contrast CT in terms of volume, local values of $\mu$, and SNR, which was defined as the ratio of the mean intensity of bone section to the standard deviation of intensity of surrounding soft (muscle) tissue. For the analysis of bone-defect repair, newly formed bone was segmented from a cylindrical region of interest, chosen centrally within the cortical defect. Bone regeneration was compared between three groups in terms of volume and $\mu$ distribution.

\section{Statistics}

Equality of variances and normality of data frequency distribution were verified by Bartlett's test and KolmogorovSmirnov test, respectively. Kruskal-Wallis tests followed by Dunn's multiple comparison tests were used to assess differences among the three groups of mice (Sham, OVX, and OVX-E) because the data frequently violated the assumptions of equality of variances between groups or Gaussian distribution. All data were analyzed by Prism 6 (GraphPad Software; San Diego, CA, USA), and $P<0.05$ was considered statistically significant.

\section{Results and discussion}

\section{Propagation-based imaging with single-distance phase retrieval versus conventional absorption imaging}

Figure 2 shows transmission X-ray images of the same proximal parts of the tibia and fibula obtained by conventional absorption imaging, propagation-based imaging, and propagation-based imaging with single-distance phase retrieval. Without phase retrieval, propagationbased imaging provides an image similar in appearance to the absorption image because the image contrast of both images is based mainly on X-ray absorption. However, the edge-enhancement effect is observed as the fringes at the boundary between bone and soft tissue, thereby allowing the clearer delineation of bone surface than in the absorption imaging. With phase retrieval, propagation-based imaging produces a high-contrast image with a considerably higher SNR compared with the other two images, owing to the effects of high X-ray sensitivity of phase shifting and low-pass filtering in Eq. 2. Instead, phase retrieval results in reduced resolution, as is evident from blurred bone boundaries in Fig. 2c compared with those in Fig. 2b.
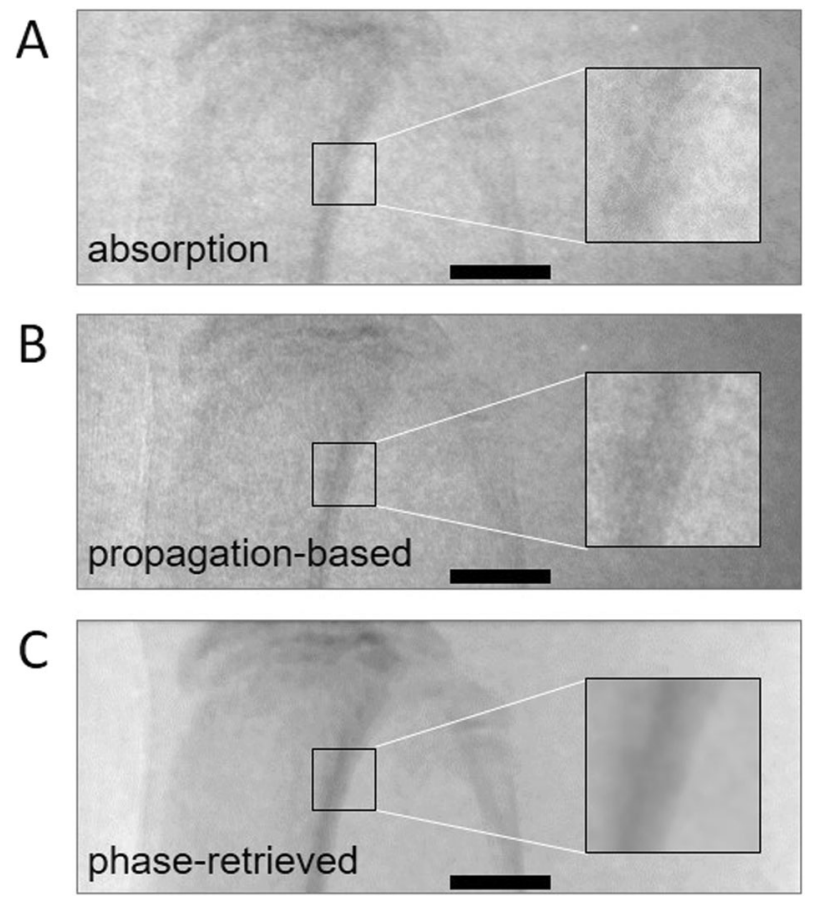

Fig. 2 a, b Transmission images acquired on the detector, located at $10 \mathrm{~cm}$ (absorption image) and $60 \mathrm{~cm}$ (propagation-based image) downstream of the object, showing the proximal part of the tibia and fibula. c Phase-retrieved image of the middle. Bar, $1 \mathrm{~mm}$

\section{PB-PCT versus absorption-contrast CT}

In Fig. 3a, axial tomographic images of the proximal tibial metaphysis are compared between PB-PCT and absorptioncontrast CT after image registration. The effect of low-pass filtering through phase retrieval appears as reduced noise and ring artifact. The visibility of trabecular and cortical porous structures is comparable between the PB-PCT and absorption-contrast CT images as previously demonstrated [23]. When bone sections were segmented with a threshold of $\mu=0.8 \mathrm{~cm}^{-1}$, values of SNR are 12.2 and 9.8 in the PBPCT and absorption-contrast CT images, respectively. The dose/scan of $6 \mathrm{~Gy}$ in absorption-contrast CT was three times higher than that of phase-contrast CT; thus, according to the relation of SNR $\propto \sqrt{\text { dose }}$ [12], SNR is estimated to decrease to 5.7 in absorption-contrast $\mathrm{CT}$ under the same dose/scan as in PB-PCT. Figure $3 \mathrm{~b}$ shows the two binarized bone sections $\left(\mu>0.8 \mathrm{~cm}^{-1}\right)$ from Fig. 3a stacked on top of each other. The bone regions observed only in PB-PCT and only in absorption-contrast $\mathrm{CT}$ amount to $5.7 \%$ and $6.6 \%$, respectively, relative to the overlapped bone region of $1.136 \mathrm{~mm}^{2}$. Indeed, 3D bone images reconstructed by PBPCT and absorption-contrast CT (Fig. 3c) show a close similarity in bone structure except for small fragments of trabecular bone. The bone regions observed only in PBPCT and only in absorption-contrast CT amount to $4.0 \%$ and $3.5 \%$, respectively, relative to the common bone region 
Fig. 3 a Tomographic bone images of tibial proximal metaphyseal cross-section reconstructed by PB-PCT and absorption-contrast $\mathrm{CT}$, matched by $3 \mathrm{D}$ image registration. The gray level (0-255) in the crosssectional images is proportional to $\mu\left(0-2.7 \mathrm{~cm}^{-1}\right)$. $\mathbf{b}$ Images in $\mathbf{a}$ are positioned on top of each other. c 3D bone images, segmented into cortical (gray) and trabecular (white) bone regions. b, c Bone regions are segmented with a threshold of $\mu=0.8 \mathrm{~cm}^{-1}$. Bars in $\mathbf{a}$ and b, $400 \mu \mathrm{m}$
A

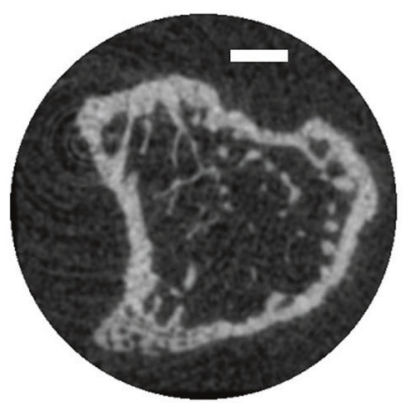

absorption-contrast CT

B

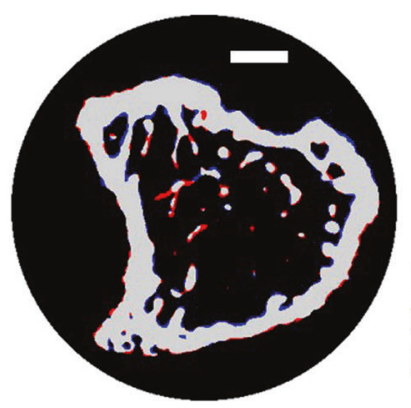

overlapped

phase-contrast CT only

absorption-contrast CT only

C

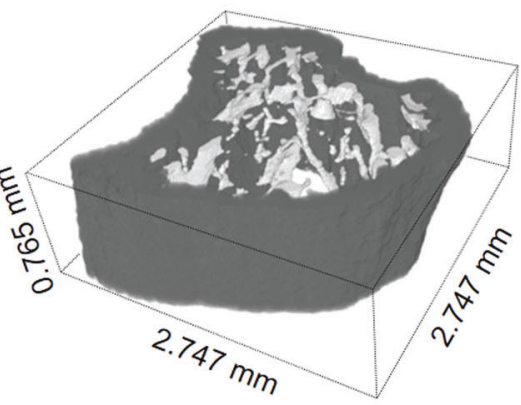

absorption-contrast CT

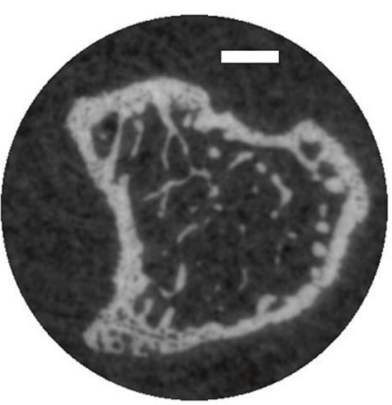

phase-contrast CT

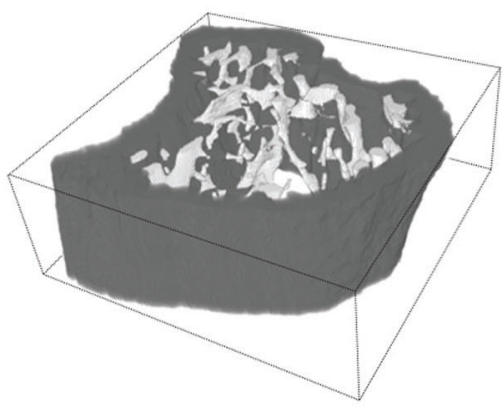

phase-contrast CT of $0.684 \mathrm{~mm}^{3}$, and the volume differences in cancellous and cortical bones between the two matched images are $4.7 \%$ and $0.3 \%$, respectively. The partial volume effect intrinsic to $\mathrm{CT}$, which is highlighted in the trabecular bone with a local thickness of only a few voxels, is mainly responsible for the larger volume difference in cancellous bone. The volume difference in cancellous bone is similar to that reported as the error of image registration in early reproducibility studies of the absorption-contrast CT quantification of trabecular bone architecture [4, 5, 41], indicating the equivalent performance of PB-PCT and absorption-contrast $\mathrm{CT}$ in delineating bone structure.

Single-distance PB-PCT was originally intended for imaging of a homogeneous object or an object with constant $\delta / \beta$ and, as such, is deemed suitable for analysis of bone architecture rather than quantification of bone density or mineralization. However, Figs. 2 and 3a suggest that some quantitative information on bone density is available from bone tomographic imaging by PB-PCT. Figure 4a shows a scatter plot of $\mu$ of the phase- versus absorption-contrast CT images in Fig. 3a; here, Gaussian blur with a radius of 1.5 pixels was applied to the latter, yielding a similar standard deviation of background (soft tissue) intensity between the two images $\left(0.12\right.$ vs. $\left.0.11 \mathrm{~cm}^{-1}\right)$. There was a significant correlation between local $\mu$ values in the matched regions in Fig. 3b (nonparametric Spearman correlation coefficient, calculated exclusively for the regions of $\mu>0.8 \mathrm{~cm}^{-1}$ ) although the regression slope deviated significantly from unity and the lines of identity and regression gradually diverge as $\mu$ decreases. Thus, PB-PCT could provide a certain amount of information on $\mu$, possibly allowing the rough assessment of bone mineralization. Note that the wide scattering of plots was partly resulted from noise or statistical uncertainty in X-ray CT imaging [42] and the image registration error. The latter could be evaluated by matching a stack of absorption-contrast CT images of tibial bone that was rotated by $3^{\circ}$ around each orthogonal axis and translated by a 1.5-voxel length along each axis. The correlation 


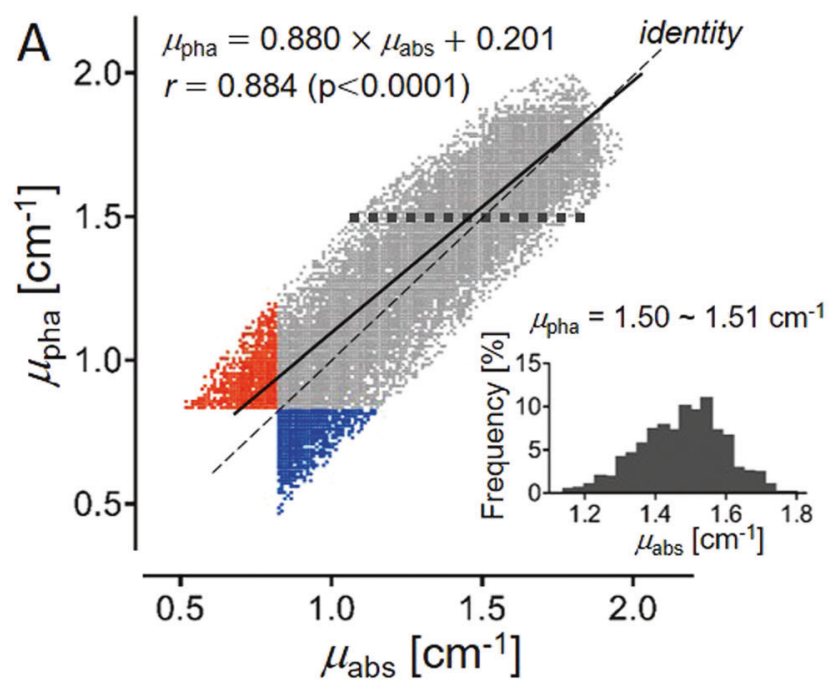

$$
\begin{aligned}
\mathrm{B} \mu_{\mathrm{abs}} & =2.282 \times \rho-1.958(\mathrm{p}<0.0001) \\
\mu_{\mathrm{pha}} & =1.885 \times \rho-1.589(\mathrm{p}<0.0001) \\
\mu_{\mathrm{pha}} & =0.826 \times \mu_{\mathrm{abs}}+0.028(\mathrm{p}<0.001)
\end{aligned}
$$

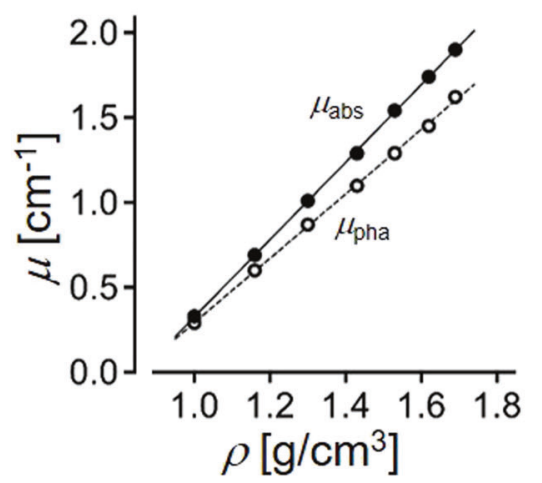

Fig. 4 a Scatter plot of $\mu$ of the phase- versus absorption-contrast CT images in Fig. 2a. The gray plots are confined to the overlapped region in Fig. 2b, where $\mu_{\text {pha }}>0.8 \mathrm{~cm}^{-1}$ and $\mu_{\mathrm{abs}}>0.8 \mathrm{~cm}^{-1}$. The red and blue plots are confined to the region where $\mu_{\mathrm{pha}}>0.8 \mathrm{~cm}^{-1}$ but $\mu_{\mathrm{abs}} \leq$ $0.8 \mathrm{~cm}^{-1}$ and vice versa, respectively, corresponding to the mismatched red and blue regions in Fig. 2b. A nonparametric Spearman correlation coefficient $(r)$ is calculated for the gray plots. Dashed line shows identity. The regression slope deviated significantly from unity

between local $\mu$ values of the matched cross-sections $(\mu>$ $\left.0.8 \mathrm{~cm}^{-1}\right)$ was less scattered with $r=0.950(P<0.0001)$.

Figure $4 \mathrm{~b}$ showed the linear relation between $\mu$ and the density of $\mathrm{K}_{2} \mathrm{HPO}_{4}$ solution in PB-PCT and absorptioncontrast CT. A threshold of $\mu=0.8 \mathrm{~cm}^{-1}$ corresponds to the solution density of $1.27 \mathrm{~g} / \mathrm{cm}^{3}$ in PB-PCT and $1.21 \mathrm{~g} / \mathrm{cm}^{3}$ in absorption-contrast $\mathrm{CT}$, and $\mu=2.0 \mathrm{~cm}^{-1}$ corresponds to the solution density of $1.90 \mathrm{~g} / \mathrm{cm}^{3}$ in PB-PCT and $1.734 \mathrm{~g} / \mathrm{cm}^{3}$ in absorption-contrast CT.

In the present study, $\gamma$ in Eq. 2 (the parameter of the Lorentzian low-pass filter) was given as $\left(\delta_{b}-\delta_{s}\right) /\left(\beta_{b}-\beta_{s}\right)$ for reducing the artifact of over-smoothing at the bone/softtissue interface. Here, we set $\gamma=3.34 \times 10^{2}$, which was derived as the value of fully-mineralized bone tissue. However, $\gamma$ varies from region to region according to the degree of local mineralization and the mineral-to-matrix ratio, and the variation of $\gamma$ within a segmented bone volume depends on the threshold of $\mu$ (see the Supplementary material). When using a threshold of $\mu=0.8 \mathrm{~cm}^{-1}$, our estimate shows that regional $\gamma$ could be higher by $7.8-47.1 \%$ than the set value (i.e., $3.34 \times 10^{2}$ ). Such differences in $\gamma$ over a segmented bone volume increase by lowering the threshold value of $\mu$, and the performance of single-distance PB-PCT will become less quantitative, especially where $\gamma$ differs to the larger extent from the set value of $\gamma$. It was already reported that PB-PCT based on the assumption of constant $\gamma$ or homogeneous object was were found to be less than quantitative when $\gamma$ varies largely
(95\% confidence limits of slope: 0.874-0.886). Inset: Histogram of $\mu_{\text {abs }}$ in the range of $\mu_{\text {pha }}=1.50-1.51 \mathrm{~cm}^{-1}$ on the horizontal dotted line (bin width: $0.03 \mathrm{~cm}^{-1}$ ). Its mean and standard deviation are 1.48 and $0.12 \mathrm{~cm}^{-1}$, respectively. b Reconstructed $\mu$ as a function of density $(\rho)$ of $\mathrm{K}_{2} \mathrm{HPO}_{4}$ solution, serving as the measure of apparent mineralization. A highly linear relationship was found between $\mu$ and the density of $\mathrm{K}_{2} \mathrm{HPO}_{4}$ solution

across reconstructed images; the image quality decreased more in regions where $\gamma$ differs further from the chosen value [43].

\section{Monitoring of bone-defect repair}

On days 3,7 , and 11 , average body weights were $20.2 \pm$ $0.75,20.8 \pm 0.41$, and $21.3 \pm 0.52 \mathrm{~g}$ in Sham; $22.4 \pm 0.98$, $22.6 \pm 0.79$, and $22.6 \pm 0.98 \mathrm{~g}$ in OVX; and $20.5 \pm 0.84$, $21.0 \pm 0.89$, and $21.7 \pm 1.03 \mathrm{~g}$ in OVX-E. No difference was found among groups on each day of CT measurements.

Newly formed bone was segmented from the cortical defect region with a threshold of $\mu=1.1 \mathrm{~cm}^{-1}$ as done in our previous studies of absorption contrast CT [44, 45]. This threshold corresponds to a density of $\mathrm{K}_{2} \mathrm{HPO}_{4}$ solution of $1.43 \mathrm{~g} / \mathrm{cm}^{3}$ in PB-PCT. Decreasing the range of bone density by setting a higher threshold of $\mu$ leads to a smaller variation of $\gamma$ across the segmented bone volume, thereby making the assumption of constant $\gamma$ more acceptable (see the the Supplementary material). Therefore, $\gamma=3.34 \times 10^{2}$ was also used in the observation of bone-defect repair process by PB-PCT. Figure 5a, b shows tomographic images of the drill-hole defect segment of tibial bone and newly formed bone in the cortical defect of a sham-operated mouse at the three time points of scanning. Images at different time points were 3D-registered, enabling us to follow the process of bone-defect healing in individual mice. Newly formed bone was nearly undetectable on day 3 
Fig. 5 a Tomographic images of the drill-hole defect segment of tibial cortical bone, reconstructed and 3D-registered for a sham-operated mouse on days 3, 7, and 11 after drill-hole surgery. b Newly formed bone in a cylindrical region (diameter, $510 \mu \mathrm{m}$; height, $170 \mu \mathrm{m}$ ) designated in the cortical defect, corresponding to each time point. c Superimposed 3D displays of matched images of the defect segment and newly formed bone within the defect acquired on day 3,7 , and 11 . Bone regions observed only on day 7 (blue) and only on day 11 (red) were resorbed and generated, respectively, over 4 days from day 7 to day 11 . Bone regions are segmented with a threshold of $\mu=1.1 \mathrm{~cm}^{-1}$
A
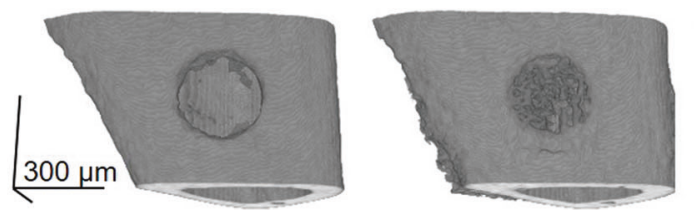

day 7

B

day 3
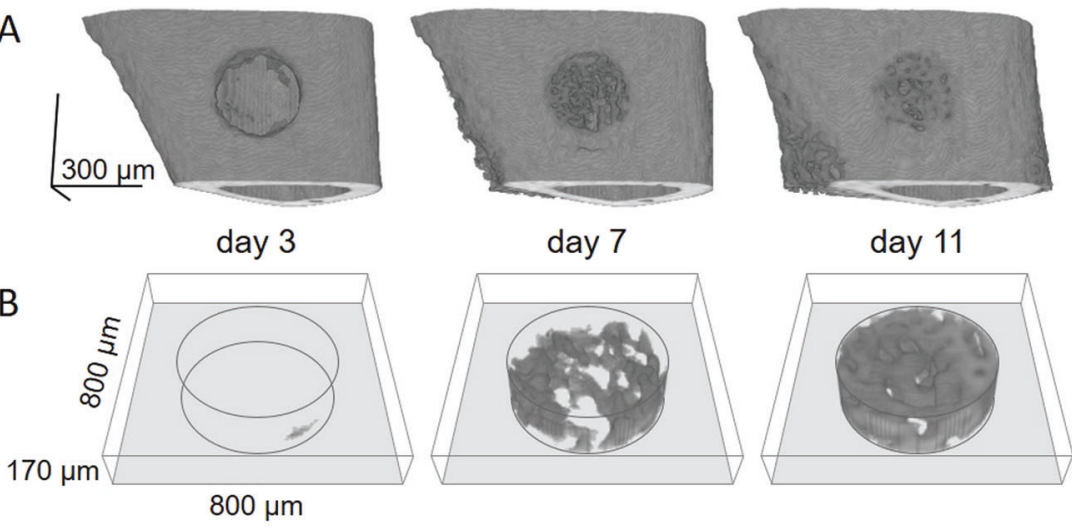

day 11

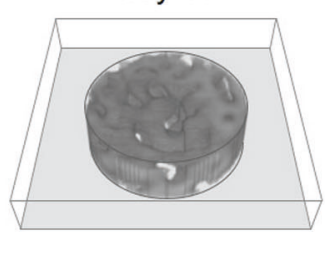

C

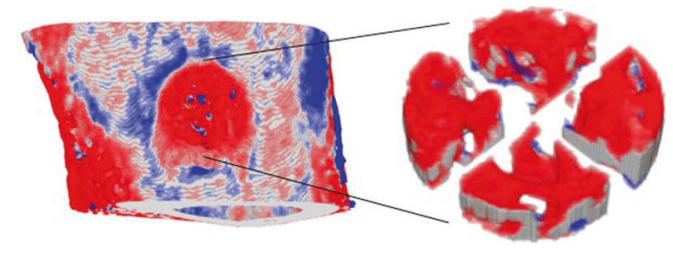

day 7 scan only

- day 11 scan only

- overlapped
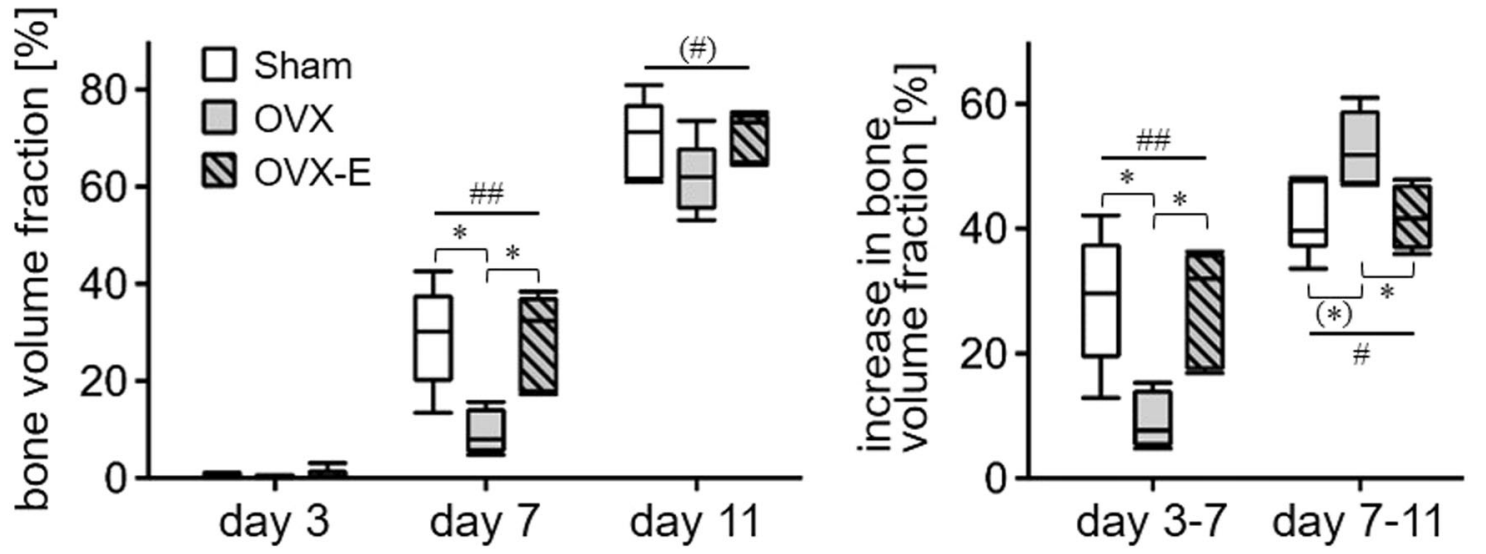

Fig. 6 Left: Volume fraction of newly formed bone in defects on days 3, 7, and 11. Right: Increase of bone volume fraction in defects over 4 days from day 3 to day 7 , and day 7 to day 11. Data are expressed as box-and-whisker plot displaying the medians, $25 \%$ quartiles, $75 \%$ quartiles, and ranges of the data. There was a significant difference $\left({ }^{\#} P<0.05,{ }^{\#} P<0.01\right)$ in bone volume fraction on day 7 and its increase over day 3 to day 7 and day 7 to day 11 or a trend of difference $\left(0.05 \leq{ }^{(\#)} P<0.1\right)$ in bone volume fraction on day 11 between groups, as assessed by Kruskal-Wallis tests. Dunn's multiple comparison post hoc tests showed that the bone volume fraction on day 7 and its increase over days 3-7 were lower in OVX than in Sham and OVX-E $\left({ }^{*} P<0.05\right)$, whereas the increase of bone volume fraction over days 7-11 was or tended to be higher in OVX than in OVX-E $\left({ }^{*} P<0.05\right)$ and Sham $\left(0.05 \leq{ }^{(*)} P<0.1\right)$, respectively because angiogenesis would be dominant at this stage $[44,45]$. On day 7 , small, thin bone segments (woven-like bone) appeared, but the large part of the defect, corresponding to void space, was still occupied by nonmineralized or low-mineralized tissues $\left(\mu<1.1 \mathrm{~cm}^{-1}\right)$. On day 11 , bone-defect repair had highly advanced; woven-like bone increased and showed a solid appearance. Figure 5c shows 3D displays of the bone defect, where the images on days 7 and 11 are registered and superimposed, giving a rough indication of local bone formation (red) with small amounts of bone resorption (blue). Over this 4-day period, new bone formation proceeded mainly outward from the already-regenerated bone (gray) and centripetally from the periphery of the defect.

In all three groups, bone-defect repair advanced similarly as shown in Fig. 5, and the defect filling reached similar levels on day 11. Some quantitative differences, however, could be detected during bone regeneration. Figure 6 shows the volume fraction of newly formed bone on days 3, 7 , and 11 and its increase over 4 days from day 3 to day 7 and day 7 to day 11. In comparison between Sham and OVX, bone regeneration was decreased in OVX over days 3-7 and consequently, the defect filling was lower in OVX on day 7, which is consistent with a previous in vivo 

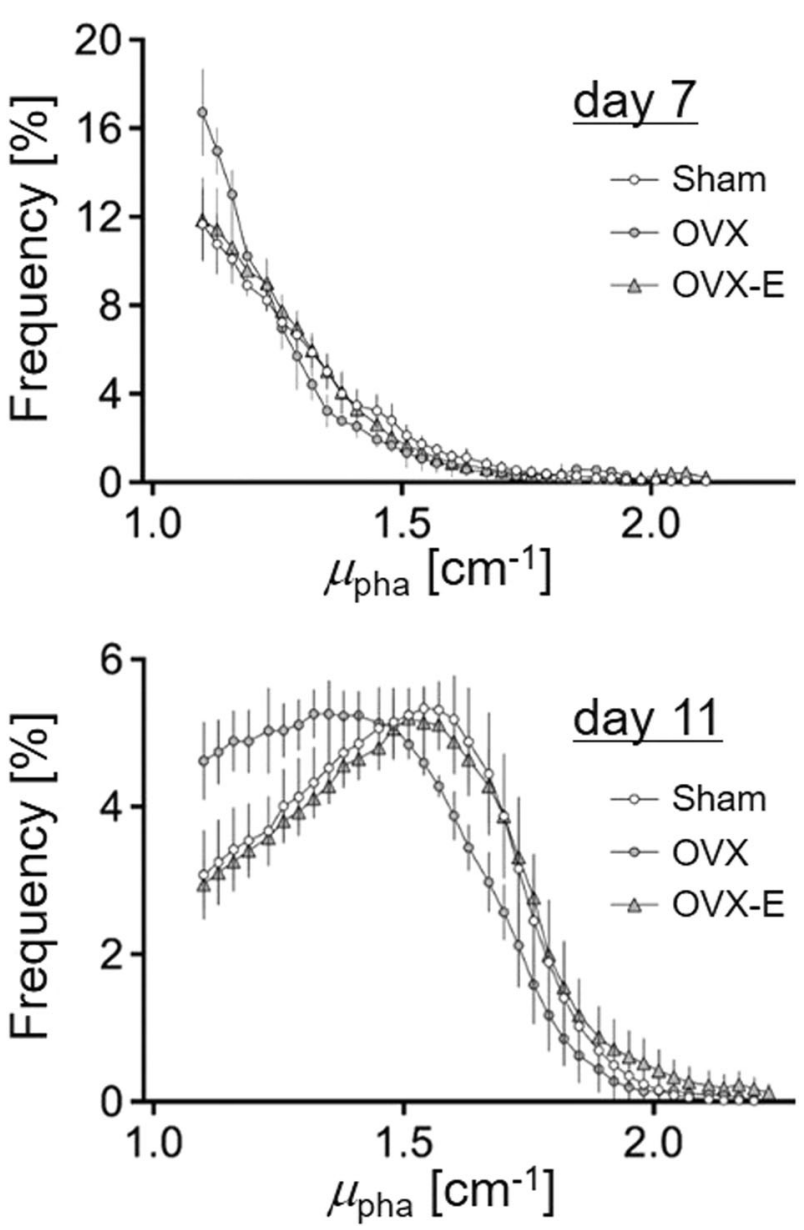

Fig. 7 Relative distribution of bone volume versus $\mu$ within the defect region obtained from Sham, OVX, and OVX-E on days 7 (top) and 11 (bottom). The bone volume at each $\mu$ value (bin width: $0.03 \mathrm{~cm}^{-1}$ is expressed as a percentage of the total volume of voxels where of $\mu>$ $1.1 \mathrm{~cm}^{-1}$. Values are mean \pm standard deviation

absorption-contrast CT study [30]. Over days 7-11, however, bone regeneration tended to advance more in OVX than in Sham, showing a similar degree of bone repair on day 11 between the two groups. No difference was found in the defect filling between Sham and OVX-E through the experimental period. Bone repair advanced more in OVX-E than in OVX over days 3-7, and accordingly, the defect filling was higher in OVX-E than in OVX on day 7. However, bone regeneration accelerated more in OVX than in OVX-E over days 7-11, leading to no difference in the defect filling on day 11 between the two groups.

Considering a significant correlation in $\mu$ values between PB-PCT and absorption-contrast CT and a proportional relation between mineral density and $\mu$ (Fig. 4), which accurately estimated by the latter under the use of monochromatic X-rays, PB-PCT would be feasible for assessment of bone mineralization, at least over the stages of bone regeneration. Figure 7 compares frequency distributions of $\mu$ (relative number of voxels at each $\mu$ value) obtained from
Sham, OVX, and OVX-E. On day 7, a monotonic decrease of frequency with increasing $\mu$ was observed in all three groups; in particular, Sham and OVX had very similar distribution. On day 11 , the frequency distribution of $\mu$ in OVX differed from that in Sham and OVX-E. At this stage, very similar monomodal distributions were observed in Sham and OVX-E with a peak each at $\mu=1.54$ and 1.51 $\mathrm{cm}^{-1}$, respectively. This shift of $\mu$ distribution from days 7 to 11 is very similar to that in a similar bone defect in mice, observed by synchrotron radiation-based absorption CT [45]. In OVX, however, no clear peak was observed and the distribution was still biased toward lower $\mu$ values, indicating that bone mineralization was delayed. As a result, on day 11, although all three groups showed similar bone regeneration in volumetric terms, the measure of mineralization of newly formed bone was low in OVX compared with the other groups. Thus, PB-PCT permits the detection of the anticipated effectiveness of 14,15-EET in improving the bone repair in OVX in terms of both volume and mineralization. Note that a measure of volume fraction of newly formed bone (Fig. 6) varies depending on a threshold of $\mu$ for bone segmentation; when it is set at a higher value by a certain extent than $1.1 \mathrm{~cm}^{-1}$, the ovariectomy-induced impairment of bone healing and its improvement by 14,15 EET will become apparent in volumetric terms on day 11.

Epoxyeicosatrienoic acids (EETs) promote regeneration of multiple organs and tissues in vivo, and this effect is partially mediated by EET-dependent stimulation of angiogenesis via vascular endothelial growth factor [28, 32]. A study using a mouse model of femoral drill-hole injury showed that bone repair was impaired and preceded by poor angiogenesis in ovariectomy-induced osteoporosis [31]. Angiogenesis is essential for bone healing; [33] thus, it is possible that the administration of 14,15-EET improves angiogenesis and thereby the bone-defect healing in OVX. Further studies are required to elucidate the pro-angiogenic effect of EETs on the improvement of bone repair. Furthermore, it was reported recently that EETs inhibit osteoclastogenesis through multiple pathways and almost completely prevented ovariectomy-induced osteoporosis [46]. Apart from the pro-angiogenic effect of EETs, there is also a need to determine whether EETs may play a direct role in promoting osteoblastogenesis.

\section{Limitations}

In repeated CT scans, the radiation effect on bone is likely to vary depending on not only cumulative dose but also on scanning protocols as well as animal species, age, and size. In 17-week-old mice, three weekly scans with $1.255 \mathrm{~Gy}$ (cumulatively $3.765 \mathrm{~Gy}$ ) had no effect on femoral trabecula [47]. Conversely, in 12-week-old mice, three-four weekly scans with 0.8459 Gy (cumulatively 2.538-3.384 Gy) 
decreased the volume fraction of trabecular bone in the radiated tibiae [10]. The same study also showed that rats were more resilient to the same amount of radiation exposure than mice [10]. Recently, the radiation effect of repeated CT scans on bone development of young rats, which are more sensitive to radiation [48], were investigated [11]. A weekly dose of $0.83 \mathrm{~Gy} / \mathrm{scan}$ (cumulatively $7.47 \mathrm{~Gy}$ ) over 8 weeks through the ages from 4 to 12 weeks did not interfere with tibial bone development. However, a weekly dose of $1.65 \mathrm{~Gy} / \mathrm{scan}$ (cumulatively $14.85 \mathrm{~Gy}$ ) or more adversely affected marrow cells and growth of tibiae. Although the radiation effects on bone regeneration could not be extrapolated from the effects on trabecula or on bone development, two doses of $2 \mathrm{~Gy} / \mathrm{scan}$ with a 3-day interval in the present study will exceed a safe level of radiation and influence bone regeneration, even though the cumulative dose (4 Gy excluding the third scan, following which of the animals are sacrificed) would stay within a safe limit. However, at least apparently, the repair of cortical bone defects occurred similarly as reported in earlier studies using a drill-hole injury model in mice [31, 45]. Monitoring over a week or so after the end of scanning might fail to detect a radiation-induced impairment of bone repair. Indeed, the effects on bone and marrow cell activities were shown to be evident at an earlier date [11, 49, 50], and the radiation effect on bone growth has been reported to reach a maximum 7-14 days after irradiation [51].

Spatial resolution and SNR in CT are limited by the radiation dose that can be applied; an increased dose is required with the fourth power of linear resolution to maintain SNR [12]. Conversely, by lowering the resolution, the dose can be reduced substantially. In the present CT system, the dose/scan actually decreases to the same level $(0.5 \mathrm{~Gy} / \mathrm{scan})$ as used in laboratory in vivo CT by increasing the voxel size from 6.54 to $9.25 \mu \mathrm{m}$. Furthermore, by allowing for the decrease in SNR to the comparable level with absorptioncontrast CT as shown in Fig. 3a, the dose could be further reduced. Multidistance phase retrieval may be a possible alternative for reducing radiation dose in PB-PCT. Under the same radiation dose, multidistance PB-PCT could result in a higher quality of image reconstruction than single-distance PB-PCT, especially with optimizing the number of distances and exposure time at each distance [52]. Although the extra time for moving a detector and taking additional reference images is undesirable from the viewpoint of animal postural maintenance, this dose-effective method would be valuable for in vivo monitoring of bone microstructure.

\section{Conclusions}

We assessed the feasibility and validity of synchrotron radiation-based PB-PCT with single-distance phase retrieval for in vivo bone imaging through its application to monitoring of bone-defect healing at three different time points in sham-operated mice, ovariectomized mice, and ovariectomized mice administered with 14,15-EET. Images of newly formed bone segmented by simple thresholding could demonstrate the ovariectomy-induced impairment of bone healing and the protective effect of 14,15-EET against it. Although imaging of bone tissue goes beyond its original use, PB-PCT with single-distance phase retrieval is shown to be potentially useful in tracking of local changes in not only bone architecture but also bone mineralization. However, its quantitative performance will be degraded for bone tissue with broad ranges of mineralization and mineral-to-matrix ratio. The future challenge is lowering the radiation dose to levels supposed to be safe, while maintaining image quality. The common dose used in bone scanning by synchrotron light lies between 0.5 and $1 \mathrm{~Gy}$ [25]. Furthermore, although the present spatial resolution could serve to track the drastic changes in bone structure, the higher spatial resolution is necessary for identifying the micro-loci of bone formation and resorption through suppressing the partial volume effect or promoting the accuracy of image registration.

Acknowledgements In vivo SRCT was performed at SPring-8 with the approval of the Japan Synchrotron Radiation Research Institute (proposal nos. 2013B1731, 2014A1725). Part of this study was supported by Grants-in-Aid for Scientific Research (grant nos. 26282120, $15 \mathrm{~K} 12509$ and 18K19926) from the Ministry of Education, Culture, Sports, Science, and Technology. The authors are indebted to Shota Sato, Yoshihiro Hashimoto, and Shinya Itamochi (Osaka University Graduate School of Engineering Science) for their assistance with animal experiments, and Shohei Hashimoto and Kazuki Ikeda (Faculty of Engineering, Tokushima University) for their assistance with image processing. We thank Libby Cone, MD, MA, from Edanz Group Japan for editing a draft of this manuscript.

\section{Compliance with ethical standards}

Conflict of interest The authors declare that they have no conflict of interest.

Publisher's note Springer Nature remains neutral with regard to jurisdictional claims in published maps and institutional affiliations.

\section{References}

1. Müller R. Hierarchical microimaging of bone structure and function. Nat Rev Rheumatol. 2009;5:373-81.

2. Kinney JH, Ryaby JT, Haupt DL, Lane NE. Three-dimensional in vivo morphometry of trabecular bone in the OVX rat model of osteoporosis. Technol Health Care. 1998;6:339-50.

3. Campbell GM, Sophocleous A. Quantitative analysis of bone and soft tissue by micro-computed tomography: applications to ex vivo and in vivo studies. Bonekey Rep. 2014;3:564.

4. Waarsing JH, Day JS, van der Linden JC, Ederveen AG, Spanjers $\mathrm{C}$, De Clerck N, et al. Detecting and tracking local changes in the 
tibiae of individual rats: a novel method to analyse longitudinal in vivo micro-CT data. Bone. 2004;34:163-9.

5. Matsumoto $T$, Nishikawa $K$, Tanaka $M$, Uesugi $K$. In vivo $C T$ quantification of trabecular bone dynamics in mice after sciatic neurectomy using monochromatic synchrotron radiation. Calcif Tissue Int. 2011;88:432-41.

6. Lukas C, Ruffoni D, Lambers FM, Schulte FA, Kuhn G, Kollmannsberger $\mathrm{P}$, et al. Mineralization kinetics in murine trabecular bone quantified by time-lapsed in vivo micro-computed tomography. Bone. 2013;56:55-60.

7. Altman AR, Tseng WJ, de Bakker CMJ, Chandra A, Lan S, Huh BK, et al. Quantification of skeletal growth, modeling, and remodeling by in vivo micro computed tomography. Bone. 2015;81:370-9.

8. Birkhold AI, Razi H, Weinkamer R, Duda GN, Checa S, Willie BM. Monitoring in vivo (re)modeling: a computational approach using 4D microCT data to quantify bone surface movements. Bone. 2015;75:210-21.

9. Pauchard Y, Mattmann C, Kuhn A, Gasser JA, Boyd SK. European society of biomechanics S.M. Perren Award 2008: Using temporal trends of 3D bone micro-architecture to predict bone quality. J Biomech. 2008;41:2946-53.

10. Klinck RJ, Campbell GM, Boyd SK. Radiation effects on bone architecture in mice and rats resulting from in vivo microcomputed tomography scanning. Med Eng Phys. 2008;30:888-95.

11. Mustafy T, Benoit A, Londono I, Moldovan F, Villemure I. Can repeated in vivo micro-CT irradiation during adolescence alter bone microstructure, histomorphometry and longitudinal growth in a rodent model? PLoS ONE. 2018;13:e0207323.

12. Ford NL, Thornton MM, Holdsworth DW. Fundamental image quality limits for microcomputed tomography in small animals. Med Phys. 2003;30:2869-77.

13. Bayat S, Apostol L, Boller E, Brochard T, Peyrin F. In vivo imaging of bone micro-architecture in mice with 3D synchrotron radiation micro-tomography. Nucl Instrum Methods Phys Res A Accel Spectrom Detect Assoc Equip. 2005;548:247-52.

14. Momose A, Fukuda J. Phase-contrast radiographs of nonstained rat cerebellar specimen. Med Phys. 1995;22:375-9.

15. Zhou SA, Brahme A. Development of phase-contrast X-ray imaging techniques and potential medical applications. Phys Med. 2008;24:129-48.

16. Bravin A, Coan P, Suortti P. X-ray phase-contrast imaging: from pre-clinical applications towards clinics. Phys Med Biol. 2013;58: R1-35.

17. Paganin D, Mayo SC, Gureyev TE, Miller PR, Wilkins SW. Simultaneous phase and amplitude extraction from a single defocused image of a homogeneous object. J Microsc. 2002;206:33-40.

18. Gureyev TE, Mayo SC, Myers DE, Nesterets Y, Paganin DM, Pogany A, et al. Refracting Röntgen's rays: Propagation-based xray phase contrast for biomedical imaging. J Appl Phys. 2009;105:102005.

19. Langer M, Cloetens P, Guigay JP, Peyrin F. Quantitative comparison of direct phase retrieval algorithms in in-line phase tomography. Med Phys. 2008;35:4556-66.

20. Arhatari BD, Gates WP, Eshtiaghi N, Peele AG. Phase retrieval tomography in the presence of noise. J Appl Phys. 2010;107:034904.

21. Gureyev T, Mohammadi S, Nesterets Y, Dullin C, Tromba G. Accuracy and precision of reconstruction of complex refractive index in near-field single-distance propagation-based phase-contrast tomography. J Appl Phys. 2013;114:144906.

22. Langer M, Liu Y, Tortelli F, Cloetens P, Cancedda R, Peyrin F. Regularized phase tomography enables study of mineralized and unmineralized tissue in porous bone scaffold. $\mathrm{J}$ Microsc. 2010;238:230-9.
23. Arhatari BD, Cooper DML, Thomas CDL, Clement JG, Peele AG. Imaging the 3D structure of secondary osteons in human cortical bone using phase-retrieval tomography. Phys Med Biol. 2011;56:5265-74.

24. Mohammadi S, Larsson E, Alves F, Dal Monego S, Biffi S, Garrovo C, et al. Quantitative evaluation of a single-distance phase-retrieval method applied on in-line phase-contrast images of a mouse lung. J Synchrotron Radiat. 2014;21:784-9.

25. Pratt I, Belev G, Zhu N, Chapman LD, Cooper DML. In vivo imaging of rat cortical bone porosity by synchrotron phase contrast micro computed tomography. Phys Med Biol. 2015;60:211-32.

26. Gasilov S, Mittone A, Horng A, Geith T, Bravin A, Baumbach T, et al. Hard X-ray index of refraction tomography of a whole rabbit knee joint: a feasibility study. Phys Med. 2016;32:1785-9.

27. Kitchen MJ, Buckley GA, Gureyev TE, Wallace MJ, Andres-Thio $\mathrm{N}$, Uesugi $\mathrm{K}$, et al. CT dose reduction factors in the thousands using X-ray phase contrast. Sci Rep. 2017;7:15953.

28. Panigrahy D, Kalish BT, Huang S, Bielenberg DR, Le HD, Yang $\mathrm{J}$, et al. Epoxyeicosanoids promote organ and tissue regeneration. Proc Natl Acad Sci USA. 2013;110:13528-33.

29. Nuzzo S, Peyrin F, Cloetens P, Baruchel J, Boivin G. Quantification of the degree of mineralization of bone in three dimensions using synchrotron radiation microtomography. Med Phys. 2002;29:2672-81.

30. Namkung-Matthai H, Appleyard R, Jansen J, Hao Lin J, Maastricht S, Swain M, et al. Osteoporosis influences the early period of fracture healing in a rat osteoporotic model. Bone. $2001 ; 28: 80-6$.

31. He YX, Zhang G, Pan XH, Liu Z, Zheng LZ, Chan CW, et al. Impaired bone healing pattern in mice with ovariectomyinduced osteoporosis: a drill-hole defect model. Bone. 2011;48:1388-400.

32. Webler AC, Michaelis UR, Popp R, Barbosa-Sicard E, Murugan A, Falck JR, et al. Epoxyeicosatrienoic acids are part of the VEGF-activated signaling cascade leading to angiogenesis. Am J Physiol Cell Physiol. 2008;295:C1292-301.

33. Carano RA, Filvaroff EH. Angiogenesis and bone repair. Drug Discov Today. 2003;8:980-9.

34. Goto S, Takeshita K, Suzuki Y, Ohashi H, Asano Y, Kimura H, et al. Construction and commissioning of a 215-m-long beamline at SPring-8. Nucl Instrum Methods Phys Res A Accel Spectrom Detect Assoc Equip. 2001;467:682-5.

35. Gureyev TE, Nesterets YI, Stevenson AW, Miller PR, Pogany A, Wilkins SW. Some simple rules for contrast, signal-to-noise and resolution in in-line $\mathrm{x}$-ray phase-contrast imaging. Opt Express. 2008; $16: 3223-41$.

36. Teague MR. Deterministic phase retrieval: a Green's function solution. J Opt Soc Am. 1983;73:1434-41.

37. Myers GR, Mayo SC, Gureyev TE, Paganin DM, Wilkins SW. Polychromatic cone-beam phase-contrast tomography. Phys Rev A. 2007;76:045804.

38. Beltran MA, Paganin DM, Uesugi K, Kitchen MJ. 2D and 3D Xray phase retrieval of multi-material objects using a single defocus distance. Opt Express. 2010;18:6423-36.

39. Beltran MA, Paganin DM, Siu KK, Fouras A, Hooper SB, Reser $\mathrm{DH}$, et al. Interface-specific x-ray phase retrieval tomography of complex biological organs. Phys Med Biol. 2011;56:7353-69.

40. Maes F, Collignon A, Vandermeulen D, Marchal G, Suetens P. Multimodality image registration by maximization of mutual information. IEEE Trans Med Imaging. 1997;16:187-98.

41. Voor MJ, Yang S, Burden RL, Waddell SW. In vivo micro-CT scanning of a rabbit distal femur: Repeatability and reproducibility. J Biomech. 2008;41:186-93.

42. Diwakar M, Kumar M. A review on CT image noise and its denoising. Biomed Signal Process Control. 2018;42:73-88. 
43. Langer M, Cloetens P, Hesse B, Suhonen H, Pacureanu A, Raum K, et al. Priors for X-ray in-line phase tomography of heterogeneous objects. Philos Trans A Math Phys Eng Sci. 2014;372:20130129.

44. Matsumoto T, Goto D, Sato S. Subtraction micro-computed tomography of angiogenesis and osteogenesis during bone repair using synchrotron radiation with a novel contrast agent. Lab Investig. 2013;93:1054-63.

45. Matsumoto T, Goto D. Effect of low-intensity whole-body vibration on bone defect repair and associated vascularization in mice. Med Biol Eng Comput. 2017;55:2257-66.

46. Guan H, Zhao L, Cao H, Chen A, Xiao J. Epoxyeicosanoids suppress osteoclastogenesis and prevent ovariectomy-induced bone loss. FASEB J. 2015;29:1092-101.

47. Judex S, Chung H, Torab A, Xie L, Rubin CT, Donahue LR, et al. Micro-CT induced radiation does not exacerbate disuse related bone loss. In: Transactions of the 51st Annual Meeting of the Orthopaedic Research Society; 2005; Washington, DC.
48. Bouxsein ML, Boyd SK, Christiansen BA, Guldberg RE, Jepsen KJ, Müller R. Guidelines for assessment of bone microstructure in rodents using micro-computed tomography. J Bone Miner Res. 2010;25:1468-86.

49. Willey JS, Lloyd SA, Robbins ME, Bourland JD, Smith-Sielicki $\mathrm{H}$, Bowman LC, et al. Early increase in osteoclast number in mice after whole-body irradiation with 2 Gy $\mathrm{X}$ rays. Radiat Res. 2008;170:388-92.

50. Dare A, Hachisu R, Yamaguchi A, Yokose S, Yoshiki S, Okano T. Effects of ionizing radiation on proliferation and differentiation of osteoblast-like cells. J Dent Res. 1997;76:658-64.

51. Engström H, Jansson JO, Engström C. Effect of local irradiation on longitudinal bone growth in the rat: a tetracycline labelling investigation. Acta Radiol Oncol. 1983;22:129-33.

52. Frachon T, Weber L, Hesse B, Rit S, Dong P, Olivier C, et al. Dose fractionation in synchrotron radiation $\mathrm{x}$-ray phase microtomography. Phys Med Biol. 2015;60:7543-66. 